\title{
PREVALENCE AND PREDICTORS OF POST-TRAUMATIC STRESS DISORDER AND INSOMNIA AMONG MEDICAL STAFF DURING CORONAVIRUS DISEASE 2019 PANDEMIC
}

\author{
By \\ Hussein SM and Ibrahim BA \\ Department of Public health, Community Medicine, Environmental and \\ Occupational Medicine, Faculty of Medicine, Suez Canal University, Ismailia, Egypt. \\ Corresponding author: Hussein SM.E mail : sara_elsaid@med.suez.edu.eg
}

\begin{abstract}
Introduction: The coronavirus disease 2019 known as (COVID-19) pandemic has a great public concern with major psychological distress, especially on the medical staff. Aim of work: The present study aimed at improving the medical staff's mental health and productivity through assessing the prevalence, risk factors, and relationship of post-traumatic stress disorder (PTSD) and insomnia among Egyptian medical staff. Materials and methods: This cross-sectional study was carried out on 390 Egyptian medical staff including physicians and nurses using web-based survey between 29th June and 22nd July 2020. The questionnaire included personal and professional questions, PTSD Checklist for DSM-5 (PCL-5), and Insomnia Severity Index. Results: The study results displayed that the prevalence of PTSD and insomnia were $55.9 \%$ and $78.7 \%$; respectively with a significant positive correlation between PTSD and insomnia scores $(\mathrm{r}=0.614, p$ value $=0.000)$. Significant predictors of PTSS were gender (female) (OR: 1.971; 95\% CI, 1.231-3.155; $\mathrm{p}<0.05$ ) and age (OR: 0.958; $95 \% \mathrm{CI}, 0.922-0.995 ; \mathrm{p}<0.05)$, worrying about getting infected (OR: $2.355 ; 95 \% \mathrm{CI}$, $1.082-5.126 ; \mathrm{p}<0.05$ ), and having insomnia (OR: 5.639; 95\% CI, 3.146-10.108; $\mathrm{p}<$ 0.05). Significant predictors of insomnia were educational level (vocational school) (OR: 8.954; 95\% CI, 2.020-39.692; p < 0.05), and having PTSD (OR: 5.602; 95\% CI, 3.135-10.013; $\mathrm{p}<0.05$ ). Conclusion: High prevalence rates of PTSD and insomnia with significant positive correlation were found among Egyptian medical staff during COVID-19 pandemic affected by significant predictors. So, urgent psychological and organizational interventions should be carried out to decrease this phenomenon.
\end{abstract}

Keywords: COVID-19, Post-Traumatic Stress, Insomnia, Medical staff and Egypt. 


\section{Introduction}

The coronavirus disease 2019 (COVID-19) is recognized as a pandemic with an increasing public health event (Lancet, 2020 and Day, 2020). It was first identified in Wuhan, China. It is a great public concern with major psychological distress, especially on the medical staff (Zhang et al., 2020a). Many actions have been done on COVID-19, including virus information, clinical features, and diagnosis have been achieved, however to date, no effective treatment is existing (Zhang et al., 2020b).

The medical staff represents the first-line fighters dealing with patients with COVID-19. Every day, they are at high risk of infection. In addition, they are exposed to long and distressing work shifts to meet health requirements (Fava et al., 2019). During the COVID-19 pandemic, health care workers were exposed to excessive burden to participate in this event. They worried about their own health and their families' health. Moreover, they are worried about infection and the safety of their colleagues in the healthcare field. They challenged loneliness and rigid expectations, which may result in stress, anger, insomnia, and anxiety related to the uncertainty of the pandemic. The media reports daily on the epidemic, especially regarding the mortality rate of frontline medical staff. The medical staff spends a lot of time each day wearing and taking off protective equipment. After shift, medical staff are-frequently surrounded by people, their families and neighbors, because the general population may not understand properly that medical staff are especially susceptible to carry the virus back home (Zhang et al., 2020a).

Hospitals are stressful workplaces because of the increased demands and complexity of most job duties, unrealistic expectations and demands from patients and their families, the unpredictable changes in person's daily work routine, and the common dealings with ethical and end of life issues. Several of these stressors could predispose medical staff to work-related psychological disorders including posttraumatic stress disorder (PTSD) and insomnia (Fava et al., 2019).

Post-traumatic stress disorder is recognized as a psychiatric disorder which occurred due to exposure to a traumatic event or extreme stressor and responded to it with fear, horror, or helplessness. Moreover, Post-traumatic Stress Symptoms (PTSS) comes after disturbing events more than the 
range of common human experiences as accidents, rape, violent physical assaults, torture, or natural disasters. It is characterized by the typical symptom pattern of persistence of trauma, relevant stimuli avoidance, intrusions, physiological hyper-arousal, and emotional numbing (Deja et al., 2006).

During the COVID-19 pandemic, a Chinese research conducted to assess PTSD among 285 residents in Wuhan and surrounding cities. It revealed that prevalence of PTSD was 7\% in highly affected areas (Liu et al., 2020). Another Singapore study among 470 health care workers showed that $7.7 \%$ of the participants have PTSS (Tan et al., 2020). Furthermore, a Chinese cross-sectional study performed during COVID-19 pandemic on 1,563 medical staffincluding frontline medical workers revealed that $36.1 \%$ of participants had insomnia (Zhang et al., 2020a).

Thus, it is clear that the medical staff's mental health needs more attention during the COVID-19 pandemic. Accordingly, PTSD and insomnia should be more investigated during this pandemic. To date, few studies were conducted to assess PTSD and insomnia among medical staff, especially in Egypt. To fill this gap, we conducted this study.

\section{Aim of work}

The present study aimed at improving the medical staff's mental health and productivity through a better understanding of post-traumatic stress disorder and insomnia by assessing the prevalence, relationship, and risk factors of post-traumatic stress disorder and insomnia among Egyptian medical staff.

\section{Materials and methods}

Study Design: It is a cross-sectional study.

\section{Place and duration of the study:} The study was performed using an online survey on medical staff of healthcare institutions including Governmental hospitals, University hospitals, Isolation hospital during the pandemic, Private hospitals, Primary health care units and centers in Egypt. The enrolled medical staff was physicians and nurses during COVID-19 pandemic. The study was conducted between 29th June and 22nd July 2020.

Study Sample: By assuming, the prevalence of PTSD among medical staff was $7.7 \%$ (Tan et al., 2020) and the prevalence of insomnia among medical staff was 36.1\% (Zhang et al., 2020a) at the level of significance of $95 \%$, the sample size was estimated by using Epi Info version 7 software. The calculated 
sample size was 354 and by adding $10 \%$ non-response rate, the sample size was 390 participants. We also ensured that this estimated sample size has been sufficient to study predictors of post-traumatic stress and insomnia. The snowball sampling technique was used to enroll the medical staff to participate in the study. Participants who filled the questionnaire were also encouraged to send the survey forward to their colleagues.

\section{Study methods:}

The study was done through using self-administered questionnaire. The questionnaire is formed of five parts:

1-Sociodemographic data included age, educational level, marital status, residence.

2-Occupational history included occupation, specialty, current workplace, and duration of employment in years.

\section{3-Questions related to the} COVID-19 included the occurrence of family or colleagues' infection, the occurrence of personal infection, availability of protective equipment in the workplace, receiving infection control training, and worrying about getting infected.

4-Assessment of post-traumatic stress disorder: The PTSD was evaluated by the Post traumatic Stress Disorder Checklist version 5 (PCL5) according to the Diagnostic and Statistical Manual of Mental Disorders, Fifth Edition criteria (DSM-5) for PTSD. The Post traumatic Stress Disorder Checklist (PCL-5) is a 20-items selfreport scale. The items of the PCL-5 agree with DSM-5 criteria for PTSD. Respondents were asked to rate how bothered they have been by each of the 20 items in the previous month on a 5point Likert scale ranging from 0 (not at all) to 4 (extremely). In general the provisional diagnosis of PTSD using PCL-5 can be determined by summing the scores for each of the 20 items and choosing cutoff point score (31-33), or by applying the DSM-5 diagnostic rule. We determined the provisional diagnosis of post-traumatic stress disorder by summing all the 20 items (range 0-80) using a cut-point score of 33 which appears to be reasonable based on recent psychometric work and results in more reliable results than the DSM5 diagnostic rule. Also, we considered the higher cut-point score in order to minimize false positives (Weathers et al., 2013 and Blevins et al., 2015)

5-Assessment of insomnia: The Insomnia Severity Index (ISI) was used to assess the severity of insomnia. 
Every item is rated on a 0 to 4 scale, and the total score ranges from 0 to 28 . A higher score indicates more severe insomnia symptoms. A total score of $\geq 8$ is recognized as having symptoms of insomnia (Bastien et al., 2001 and Morin et al., 2011).

The questionnaire was translated to Arabic language from the original English version by using back to back translation method.

\section{Data collection}

The prepared questionnaire was converted to web-based form by Google Form Application to be filled online. A pilot study was conducted on 30 participants not included in the sample before beginning the actual data collection. The purpose of the pilot study was to ascertain the clarity, and applicability of the study tool. It also helped to estimate the time needed to fill in the questionnaire. Based on the received feedback we modified the questionnaire.

The link of the questionnaire was sent to the medical staff through emails, messenger, Whats'App messages, and Facebook groups. We searched all Egyptian medical staff groups in Facebook, entered it and embedded the questionnaire Google form to cover medical staff in all Egypt. Moreover, we invited the medical staff who responded to share the questionnaire link with their colleagues and other medical groups on social media. So, we covered as possibly all regions of Egypt.

\section{Consent}

The informed consent was appended to the questionnaire. Each questionnaire contained the informed consent in its first part. The participant had to check "I agree" to start to fulfill the questionnaire.

\section{Ethical approval}

The study was reviewed and approved by the Research Ethics Committee of the Faculty of Medicine, Suez Canal University (code 4192\#, Date: 29/6/2020).

\section{Data management}

Data entry and statistical analysis were done using the Statistical Package for Social Science (SPSS) software program version 22. The descriptive statistics were applied in the form of tables and figures as appropriate. Data was analyzed; normality of continuous variables was checked by the Shapiro-Wilk test, student t-test was used for quantitative normally distributed variables, and Mann Whitney U test was used for not normally 
distributed variables. The Chi-square and insomnia. Logistic regression analysis test was used for qualitative variables. was used to determine the predictors of postSpearman correlation was used to assess the traumatic stress and insomnia. Statistical relationship between post-traumatic stress significance was set at $\mathrm{p}<0.05$.

\section{Results}

\section{Table 1: Descriptive statistics of the medical staff's demographic and professional characteristics.}

\begin{tabular}{|l|c|c|}
\hline Variables (No=390) & Frequency & \% \\
\hline Gender & & 33.8 \\
\hline Male & 132 & 66.2 \\
\hline Female & 258 & \\
\hline Age /years, Mean(SD) & $32.02(6.78)$ & \\
\hline Marital status & & 36.7 \\
\hline Single & 143 & 59.5 \\
\hline Married & 232 & 0.8 \\
\hline Widow & 3 & 3.1 \\
\hline Divorced & 12 & \\
\hline Educational Level & & 14.1 \\
\hline Vocational school & 55 & 38.5 \\
\hline Bachelor degree & 150 & 34.4 \\
\hline Master degree & 134 & 13.1 \\
\hline Doctorate degree & 51 & 80.3 \\
\hline Occupation & & 19.7 \\
\hline Physician & 313 & 28.2 \\
\hline Nurse & 77 & 33.1 \\
\hline Workplace & & 6.4 \\
\hline Governmental hospital & 110 & 5.6 \\
\hline University hospital & 129 & 5.1 \\
\hline Isolation hospital during the pandemic & 25 & 21.5 \\
\hline Private hospital & 22 & \\
\hline Others & 20 & \\
\hline More than 1 workplace & & \\
\hline Working years, Mean(SD) & & \\
\hline
\end{tabular}

SD: Standard deviation. 
Table 2: Post-traumatic stress disorder and insomnia among study participants (No =390).

\begin{tabular}{|c|c|c|}
\hline Variables & No. & $\%$ \\
\hline \multicolumn{3}{|l|}{ Presence of PTSD } \\
\hline NO & 172 & 44.1 \\
\hline Yes & 218 & 55.9 \\
\hline \multicolumn{3}{|l|}{ Presence of insomnia } \\
\hline NO & 83 & 21.3 \\
\hline Yes & 307 & 78.7 \\
\hline \multicolumn{3}{|l|}{ Grades of insomnia } \\
\hline NO clinically significant insomnia & 83 & 21.3 \\
\hline Sub threshold insomnia & 188 & 48.2 \\
\hline Clinical insomnia (moderate severity) & 99 & 25.4 \\
\hline Clinical insomnia (severe) & 20 & 5.1 \\
\hline Score of PTSD, Mean(SD) & \multirow{2}{*}{\multicolumn{2}{|c|}{$\begin{array}{c}36.06(17.6) \\
11.96(5.7)\end{array}$}} \\
\hline Score of insomnia, Mean(SD) & & \\
\hline Spearman>s rho Correlation between PTSD and insomnia & $\mathrm{r}=0.614 \%$ & $\mathrm{le}(0.000)$ \\
\hline
\end{tabular}

PTSD: Post traumatic stress disorder,

SD: Standard Deviation,

*: Statistical significance was set at $\mathrm{p}<0.05$.

Regarding PTSD and insomnia, as presented in Table 2, the prevalence of PTSD among study participants were $55.9 \%$. But the prevalence of insomnia was $78.7 \%$. By using ISI scale to classify insomnia into grades, the proportion of medical staff having no clinically significant insomnia, sub threshold insomnia, clinical insomnia (moderate severity), and clinical insomnia (severe) were $21.3 \%, 48.2 \%, 25.4 \%$, $25.4 \%$, and 5.1\%; respectively. The mean score of PCL-5 scale of PTSD was 36.06 \pm 17.6. Additionally, the mean score of insomnia by using ISI scale was $11.96 \pm$ 5.7. With regards to the association between PTSD and insomnia, Table 2 showed a significant positive correlation between PTSD and insomnia scores by using spearman's rho test $(\mathrm{r}=0.614$, $\mathrm{p}$ value $=0.000)$. 
Table 3: Univariate analysis of risk factors of post-traumatic stress disorder.

\begin{tabular}{|c|c|c|c|}
\hline \multirow{3}{*}{ Variables $(\mathrm{No}=\mathbf{3 9 0})$} & \multicolumn{3}{|c|}{ PTSD } \\
\hline & $\begin{array}{c}\text { NO } \\
(\text { No }=172) \\
\text { No }(\%)\end{array}$ & $\begin{array}{c}\text { Yes } \\
(\text { No }=\mathbf{2 1 8}) \\
\text { No }(\%)\end{array}$ & \multirow{4}{*}{$\begin{array}{l}X^{2}(p \text { value }) \\
7.593\left(0.006^{*}\right)\end{array}$} \\
\hline & & & \\
\hline Male & $71(41.3)$ & $61(28)$ & \\
\hline Female & $101(58.7)$ & 157(72) & \\
\hline Age (years), median(IQR) & $31.5(9)$ & $30(8.25)$ & $(0.026 * a)$ \\
\hline \multicolumn{3}{|l|}{ Marital status } & \multirow{4}{*}{$1.755(0.416)$} \\
\hline Single & $64(37.2)$ & $79(36.2)$ & \\
\hline Married & $99(57.6)$ & $133(61)$ & \\
\hline Widow or divorced & $9(5.2)$ & $6(2.8)$ & \\
\hline \multicolumn{3}{|l|}{ Educational Level } & \multirow{5}{*}{$6.887(0.076)$} \\
\hline Vocational school & $22(12.8)$ & $33(15.1)$ & \\
\hline Bachelor degree & $61(35.5)$ & $89(40.8)$ & \\
\hline Master degree & $58(33.7)$ & $76(34.9)$ & \\
\hline Doctorate degree & $31(18)$ & $20(9.2)$ & \\
\hline \multicolumn{3}{|l|}{ Occupation } & \multirow{3}{*}{$1.085(0.581)$} \\
\hline Physician & $142(82.6)$ & $171(78.4)$ & \\
\hline Nurse & $30(17.4)$ & $47(21.6)$ & \\
\hline Working years, median(IQR) & $7(10)$ & $6(9)$ & $\left(0.027^{* a}\right)$ \\
\hline Occurrence of family or colleagues infection(yes) & $131(76.2)$ & $175(80.3)$ & $0.962(0.327)$ \\
\hline Occurrence of personal infection (yes) & $18(10.5)$ & 26(11.9) & $0.205(0.651)$ \\
\hline Receiving infection control training (yes) & $57(33.1)$ & $53(24.3)$ & $3.700(0.054)$ \\
\hline $\begin{array}{l}\text { Availability of protective equipment in workplace } \\
\text { (yes) }\end{array}$ & $73(42.4)$ & $82(37.6)$ & $0.935(0.333)$ \\
\hline Worrying about getting infected (yes) & $148(86)$ & 204(93.6) & $6.201(0.013 *)$ \\
\hline Insomnia (yes) & $109(63.4)$ & 198(90.8) & $43.255(0.000 *)$ \\
\hline
\end{tabular}


In the univariate analysis by using Mann Whitney $U$ and chi-square tests for risk factors of PTSD, Table 3 showed that being female $(\mathrm{p}=0.006)$, younger age $(\mathrm{p}=0.026)$, lesser years of working $(\mathrm{p}=0.027)$, worrying about getting infected $(\mathrm{p}=0.013)$, and having insomnia $(\mathrm{p}=0.000)$ were statistically significant risk factors.

\section{Table 4: Univariate analysis of risk factors of insomnia.}

\begin{tabular}{|c|c|c|c|}
\hline \multirow[b]{2}{*}{ Variables $(\mathbf{N o}=\mathbf{3 9 0})$} & \multicolumn{3}{|c|}{ Insomnia } \\
\hline & $\begin{array}{c}\text { NO } \\
(\mathrm{No}=172) \\
\text { No }(\%) \\
\end{array}$ & $\begin{array}{c}\text { Yes } \\
(\text { No }=218) \\
\text { No }(\%) \\
\end{array}$ & $\mathrm{X}^{2}$ (P value) \\
\hline \multicolumn{3}{|l|}{ Gender } & \multirow{3}{*}{$0.56(0.812)$} \\
\hline Male & $29(34.9)$ & $103(33.6)$ & \\
\hline Female & $54(65.1)$ & $204(66.4)$ & \\
\hline Age (years), median(IQR) & $32(10)$ & $30(9)$ & $\left(0.345^{\mathrm{a}}\right)$ \\
\hline \multicolumn{3}{|l|}{ Marital status } & \multirow{4}{*}{$2.051(0.359)$} \\
\hline Single & $36(43.4)$ & $107(34.9)$ & \\
\hline Married & $44(53)$ & $188(61.2)$ & \\
\hline Widow or divorced & $3(3.6)$ & $12(3.9)$ & \\
\hline \multicolumn{3}{|l|}{ Educational Level } & \multirow[t]{5}{*}{$14.929(0.002 *)$} \\
\hline Vocational school & $8(9.6)$ & $47(15.3)$ & \\
\hline Bachelor degree & $31(37.3)$ & $119(38.8)$ & \\
\hline Master degree & $23(27.7)$ & $111(36.2)$ & \\
\hline Doctorate degree & $31(25.3)$ & $30(9.8)$ & \\
\hline \multicolumn{3}{|l|}{ Occupation } & \multirow[t]{3}{*}{$1.86(0.666)$} \\
\hline Physician & $68(81.9)$ & $245(79.8)$ & \\
\hline Nurse & $15(18.1)$ & $62(20.2)$ & \\
\hline Working years, median(IQR) & $7(10)$ & $6(8)$ & $\left(0.305^{\mathrm{a}}\right)$ \\
\hline $\begin{array}{l}\text { Occurrence of family or colleagues } \\
\text { infection(yes) }\end{array}$ & $64(77.1)$ & $242(78.8)$ & $0.114(0.735)$ \\
\hline Occurrence of personal infection (yes) & $8(9.6)$ & $36(11.7)$ & $0.285(0.594)$ \\
\hline Receiving infection control training (yes) & $26(31.3)$ & $84(27.4)$ & $0.507(0.479)$ \\
\hline $\begin{array}{l}\text { Availability of protective equipment in } \\
\text { workplace (yes) }\end{array}$ & $39(47)$ & $116(37.8)$ & $2.311(0.128)$ \\
\hline Worrying about getting infected (yes) & $70(84.3)$ & $282(91.9)$ & $4.201(0.040 *)$ \\
\hline PTSD (yes) & $20(24.1)$ & $198(90.8)$ & $43.255(0.000 *)$ \\
\hline
\end{tabular}

PTSD: Post traumatic stress disorder, IQR: Inter Quartile Range, a : Mann whitney u test,

$*$ : Statistical significance at $\mathrm{p}<0.051$. 
Univariate analysis for risk factors of insomnia summarizes that educational level $(\mathrm{p}=0.002)$, worrying about getting infected $(\mathrm{p}=0.040)$, and having PTSD $(p=0.000)$ were statistically significant risk factors of insomnia as shown in Table 4.

Table 5: Multivariate logistic regression analysis of post-traumatic stress disorder and insomnia related factors.

\begin{tabular}{|c|c|c|c|c|c|}
\hline \multirow{3}{*}{ Variables $(\mathrm{No}=390)$} & \multicolumn{5}{|c|}{ Post-Traumatic Stress Disorder } \\
\hline & \multirow{2}{*}{$\boldsymbol{B}$} & \multirow{2}{*}{ p value } & \multirow{2}{*}{ OR } & \multicolumn{2}{|c|}{$95 \% \mathrm{CI}$} \\
\hline & & & & Lower & Upper \\
\hline Gender (female) & 0.678 & $0.005 *$ & 1.971 & 1.231 & 3.155 \\
\hline Age (years) & -0.043 & $0.026 *$ & 0.958 & 0.922 & 0.995 \\
\hline Educational Level (ref. doctorate) & & 0.936 & & & \\
\hline Vocational school & -0.078 & 0.909 & 0.925 & 0.243 & 3.525 \\
\hline Bachelor & 0.111 & 0.793 & 1.118 & 0.488 & 2.561 \\
\hline Master & 0.197 & 0.605 & 1.218 & .578 & 2.567 \\
\hline Occupation (nurse) & 0.670 & 0.185 & 1.954 & 0.725 & $5.264 \mathrm{UU}$ \\
\hline \multirow{2}{*}{$\begin{array}{l}\text { Worrying about getting infected (yes) } \\
\text { Presence of insomnia (yes) }\end{array}$} & 0.857 & $0.031 *$ & 2.355 & 1.082 & 5.126 \\
\hline & 1.730 & $0.000 *$ & 5.639 & 3.146 & 10.108 \\
\hline \multirow{3}{*}{ Variables $(\mathrm{No}=\mathbf{3 9 0})$} & \multicolumn{5}{|c|}{ Insomnia } \\
\hline & \multirow[t]{2}{*}{$\boldsymbol{B}$} & \multirow[t]{2}{*}{$p$ value } & \multirow[t]{2}{*}{$\mathbf{O R}$} & \multicolumn{2}{|c|}{$95 \% \mathrm{CI}$} \\
\hline & & & & Lower & Upper \\
\hline Gender (female) & -0.155 & 0.591 & 0.856 & 0.485 & 1.510 \\
\hline Age (years) & 0.023 & 0.358 & 1.023 & 0.975 & 1.073 \\
\hline Educational Level (ref. doctorate) & & $0.007 *$ & & & \\
\hline Vocational school & 2.192 & $0.004 *$ & 8.954 & 2.020 & 39.692 \\
\hline Bachelor & 1.109 & $0.017 *$ & 3.031 & 1.216 & 7.553 \\
\hline Master & 1.184 & $0.004 *$ & 3.268 & 1.470 & 7.266 \\
\hline Occupation (nurse) & -0.784 & 0.142 & 0.457 & 0.160 & 1.301 \\
\hline Worrying about getting infected (yes) & 0.548 & 0.183 & 1.730 & 0.773 & 3.873 \\
\hline Presence of PTSD (yes) & 1.723 & $0.000 *$ & 5.602 & 3.135 & 10.013 \\
\hline
\end{tabular}

PTSD: Post traumatic stress disorder,

CI: confidence interval,

OR: odds ratio.

*: Statistical significance was set at $\mathrm{p}<0.05$. 
The logistic regression analysis of risk factors of PTSD and insomnia are shown in Table 5. The significant predictors of PTSD, after controlling for confounders, were gender (female) (OR: 1.971; 95\% CI, 1.231-3.155; $\mathrm{p}<0.05$ ), age (OR: 0.958; 95\% CI, 0.922-0.995; $\mathrm{p}<0.05$ ), worrying about getting infected (OR: 2.355; 95\% CI, 1.082-5.126; $\mathrm{p}<0.05$ ), and having insomnia (OR: 5.639; 95\% CI, 3.146-10.108; $\mathrm{p}<0.05$ ). Regarding insomnia, the educational level (vocational school) (OR: 8.954; 95\% CI, 2.020-39.692; p < 0.05), bachelor degree (OR: 3.031; 95\% CI, 1.2167.553), Master (OR: 3.268; 95\% CI, 1.470-7.266), and having PTSD (OR: 5.602; $95 \%$ CI, 3.135-10.013; $p<0.05)$ were significant predictors of insomnia.

\section{Discussion}

The COVID-19 pandemic is considered recently as one of the most stressful events all over the world. The COVID-19 pandemic has also the potential to affect the mental health of healthcare workers significantly causing psychological distress, posttraumatic stress symptoms, and insomnia (Jahrami et al., 2020).

The current study aimed to assess PTSD and insomnia among Egyptian medical staff during the COVID-19 pandemic. The prevalence of posttraumatic stress disorder among the medical staff in the present study was high $(55.9 \%)$ (Table2). This high prevalence among the Egyptian medical staff may be attributed to feeling vulnerable and at risk of having infected; fear of transmitting the disease to families, colleagues, and loved ones; overwhelming situations resulting from the unexpected incidence of COVID-19 pandemic with lack of resources, heavy workloads created by the pandemic; caring for patients who are very ill and rapidly deteriorating and caring for colleagues who have also fallen sick; inadequate family, community and social support; reduced mental preparedness, and reduced accessibility to needed psychological support (Koh, 2020 and Walton et al., 2020).

Likewise, an Italian study was conducted to assess the psychological effect of the COVID-19 on a group of healthcare workers revealed that the prevalence rate of post-traumatic stress disorder was $26.2 \%$ (Di Tella et al., 2020). Also in China, Huang et al. (2020) investigated the mental health of medical staff in a tertiary infectious disease hospital for COVID-19 and reported that the prevalence of PTSD in medical staff was $27.39 \%$. 
Another Chinese cross-sectional online survey carried out on health care workers combating for the COVID-19 outbreak reported that the prevalence of PTSD one month after the COVID-19 outbreak was 3.8\% (Yin et al., 2020). Moreover, in Singapore, a crosssectional study showed that $7.7 \%$ of health care workers screened positive for clinical concern of PTSD during the COVID-19 pandemic (Tan et al., 2020). According to a study conducted in Turkey, frontline health care workers who deal with the COVID-19 patients reported also a high level of secondary post-traumatic stress symptoms (Arpacioglu et al., 2020).

The variation in the prevalence of PTSD across the countries may be attributed to the variability of the nature of the health system, the culture, the surrounding circumstances of medical staff and their attitudes that could be unique for these countries, the country-specific diagnostic and therapeutic guidelines of PTSD as well as the availability of psychological support. There are also variations in the assessment scales and cut-off scores of PTSD adopted by each study.

Moreover, the present study demonstrated that medical staff with PTSD was significantly younger and had a significantly shorter duration of employment compared to those without PTSS (Table 3). Also, the logistic regression revealed that the age was a significant predictor of PTSD (OR: 0.958; 95\% CI, 0.922-0.995; p $<0.05$ ) (Table5). A similar finding has been identified by the study done by Arpacioglu et al. (2020) who found that being in the first years of the work is associated with high secondary posttraumatic stress symptoms and also the age is independent risk factors for secondary post-traumatic stress symptoms.

Also, the result of the present study provides supporting evidence that there is a positive correlation between posttraumatic stress and insomnia $(\mathrm{r}=0.614, \mathrm{p}$ value $=0.000)$ (Table 2$)$. Moreover, the multivariate analysis of variables affecting post-traumatic stress disorder revealed that gender and specifically being female (OR: 1.971; 95\% CI, 1.231-3.155; $\mathrm{p}<0.05)$, as well as worrying about being infected (OR: 2.355; 95\% CI, 1.082-5.126; $\mathrm{p}<$ 0.05 ), and having insomnia (OR: 5.639; 95\% CI, 3.146-10.108; $\mathrm{p}<0.05)$ were significant predictors of PTSD among the study population (Table 5). These findings were consistent with previous studies that showed a higher prevalence 
of PTSD among females compared to males with statistically significant difference, in both healthcare workers and the general population (Di Tella et al., 2020 and Liu et al., 2020). Also Yin et al. (2020) found that female healthcare workers fighting for the COVID-19 were more vulnerable to PTSD. This finding may be explained by the idea that the basis of a specific vulnerability to psychological disorders in females could be attributed to variations in hormone levels which are responsible for altered sensitivity to emotional stimuli during certain phases in the menstrual cycle in which the intrusive flashbacks were enhanced (Soni et al., 2013). This agreed with Arpacioglu et al. (2020) study which indicated that both anxiety about the COVID-19 and being a woman, were associated with high secondary PTSD, and concluded that female health care workers are affected by the pandemic more than others. Furthermore, the finding of the present study was consistent with that of Yin et al. (2020) who concluded that health care workers with PTSD had poorer overall sleep quality. This pattern of results was similar with the previous research indicating that insomnia has been linked to both the onset and maintenance of PTSD and that it is not simply a secondary symptom but rather is a risk factor for PTSD. That is, poor sleep quality may result in a context and environment in which individuals are more prone to avoid trauma reminders. Accordingly, when reminders cannot be avoided, the individuals with elevated levels of anxiety and alertness can be at a great risk to develop PTSD (Babson and Feldner, 2010 and Wright et al., 2011).

Furthermore, the current work showed a high prevalence of insomnia among medical staff during the COVID-19 pandemic in Egypt (78.7\%) (Table 2). Previous studies have indicated that the medical workers were vulnerable to sleep disturbances in the ordinary times (Kaneita and Ohida, 2011 and Qiu et al., 2020). Accordingly, the medical staff personnel during the COVID-19 pandemic were more susceptible to sleep disorders. This may be due to worries related to the contagious nature of the COVID-19 and associated stress; the emergency nature of work during the COVID- 19 pandemic which may disturb sleep; increased workload and shift work with the subsequent circadian disturbance (Zhang et al., 2020a).

Similarly, a cross-sectional study was conducted 8 weeks after the 
COVID-19 epidemic outbreak in Wuhan, China to assess insomnia among health care workers showed that the prevalence of insomnia was 38.4\% (Zhang et al., 2020b).

Another Chinese study showed that $36.1 \%$ of medical staff suffered from insomnia among the 1,563 participants recruited from hospitals during the COVID-19 outbreak (Zhang et al., 2020a). Likewise, a Chinese cross-sectional survey enrolling 1257 health care workers revealed a high prevalence of insomnia (34.0\%) among health care workers treating patients with COVID-19 (Lai et al., 2020). The present work agreed with Qi et al. (2020) study which indicated that $45.5 \%$ of medical workers in Hubei Province, China during COVID-19 outbreak had insomnia according to the Athens Insomnia Scale (AIS).

Moreover, in Arab countries, a study from Bahrain, addressed sleep quality for frontline healthcare workers during the outbreak of COVID-19 and reported that $75 \%$ of healthcare workers had poor sleep quality using the Pittsburgh Sleep Quality Index (PSQI) (Jahrami et al., 2020). Also, a study conducted in Iraqi Kurdistan found that $68.3 \%$ of the physicians were sleepless during the COVID-19 pandemic using the Athens
Insomnia Scale (Abdulah and Musa, 2020).

Additionally, the present study demonstrated that more than half of the medical staff reporting symptoms of insomnia were significantly more likely to have an education level of Bachelor's degree or below $(\mathrm{p}=0.002)$ (Table 4). As well, the logistic regression analysis showed that the educational level was a negative significant predictor of the insomnia (Table 5). This agreed with the work done by Zhang and his colleagues (2020a) which indicated that medical staff in the insomnia group were more likely to have a high school education and a bachelor's degree ( $\mathrm{p}=$ 0.002). Also, the findings of this study agreed with that of Xiang et al. (2008), who conducted an insomnia survey on the Chinese general population and indicated that a low education level was significantly associated with a higher probability of insomnia. It could be that a low education level is more correlated with greater occupational stress and thus insomnia.

According to the current study, it was observed that worry about getting infected by COVID-19 was also a risk factor of insomnia (Table 4). This corresponds with what Zhang et al. (2020a) who found that medical staff 
reporting symptoms of insomnia during COVID-19 pandemic were more worried about being infected compared with those in the non-insomnia group $(p<0.001)$. Also, Kirwan et al. (2017) found, that worries among medical staff could affect sleep quality.

Finally, the logistic regression analysis in this study showed that having PTSD symptoms was a significant predictor of insomnia (OR: 5.602; 95\% CI, 3.135-10.013; $\mathrm{p}<0.05$ ) (Table 5). The result is concomitant with the finding of Yin et al. (2020) who performed a study on health care workers fighting for the COVID-19 in different provinces of China. They concluded that poor sleep quality was significantly associated with PTSD. Likewise, Taylor et al. (2016) found that PTSD was a positive significant predictor of insomnia.

\section{Conclusion}

In conclusion, high prevalence rates of post-traumatic stress disorder $(55.9 \%)$ and insomnia (78.7\%) with significant positive correlation were found among Egyptian medical staff during COVID-19 pandemic. The age, gender, worrying about being infected, and having insomnia were significant predictors for PTSD. Educational level and having PTSD symptoms was a significant predictor of insomnia.

\section{Recommendations:}

The results of the current study suggest that PTSD as well as insomnia should be considered as occupational health concerns and highlight the urgent need to implement psychological and organizational interventions to promote the mental health of medical staff. These findings also necessitate performing interventional studies to develop and test workplace interventions to reduce occupational stress and prevent development of any mental disorders including PTSD and sleep disorders.

\section{Conflict of interest}

The authors declared that there was no conflict of interest.

\section{Funding}

No financial support was received for this study.

\section{References}

1. Abdulah DM and Musa DH (2020): Insomnia and stress of physicians during COVID-19 outbreak. Sleep Med X;2(100017):1-7. doi:10.1016/j.sleepx.2020.100017

2. Arpacioglu S, Gurler $M$ and Cakiroglu S (2020): Secondary Traumatization Outcomes and Associated Factors Among the Health Care Workers Exposed to the COVID-19. Int J Soc Psychiatry;1:6. doi:10.1177/0020764020940742

3. Bastien ÂH, Vallie A and Morin CM (2001): 
Validation of the Insomnia Severity Index ( ISI) as an outcome measure for insomnia research Validation of the Insomnia Severity Index as an outcome measure for insomnia research. Sleep Med; 2(4):297-307. doi:10.1016/S13899457(00)00065-4

4. Blevins CA, Weathers FW, Davis MT, Witte TK and Domino JL (2015): The Posttraumatic Stress Disorder Checklist for DSM-5 ( PCL5 ): Development and Initial Psychometric Evaluation. J Trauma Stress; 5:489-98. doi:https://doi.org/10.1002/jts. 22059

5. Day M (2020): Covid-19: Surge in cases in Italy and South Korea makes pandemic look more likely. BMJ;368: 751. doi:10.1136/bmj. $\mathrm{m} 751$

6. Deja M, Denke C, Weber-carstens S, Schröder J, Pille C E, et al (2006): Social support during intensive care unit stay might improve mental impairment and consequently healthrelated quality of life in survivors of severe acute respiratory distress syndrome. Crit Care;10(5):1-12. doi:10.1186/cc5070

7. Di Tella M, Romeo A, Benfante A and Castelli L (2020): Mental health of healthcare workers during the COVID-19 pandemic in Italy. J Eval Clin Pract;1-5. doi:10.1111/jep.13444.

Available at: https://onlinelibrary.wiley.com/ doi/epdf/10.1111/jep.13444

8. Fava GA, Mcewen BS, Guidi J, Gostoli S, Offidani E, and Sonino N (2019): Clinical characterization of allostatic overload. Psychoneuroendocrinology; 108:94-101. doi:10.1016/j.psyneuen.2019.05.028

9. Huang JZ, Han MF, Luo TD, Ren AK and Zhou XP (2020): Mental health survey of medical staff in a tertiary infectious disease hospital for COVID-19. Chinese $\mathrm{J}$ Ind Hyg Occup Dis;38(3):192-5. doi: 10.3760/ cma.j.cn121094-20200219-00063.

10. Jahrami H, Bahammam AS, Algahtani $H$, Ebrahim A, Faris M, et al. (2020): The examination of sleep quality for frontline healthcare workers during the outbreak of
COVID-19. Sleep Breath;1-9. doi:https://doi. org/10.1007/s11325-020-02135-9

11. Kaneita Yand Ohida T(2011): Association of Current Work and Sleep Situations with Excessive Daytime Sleepiness and Medical Incidents among Japanese Physicians. J Clin sleep Med;7(5):512-22. doi:10.5664/ JCSM. 1322

12. Kirwan M, Pickett SM and Jarrett NL (2017): Emotion regulation as a moderator between anxiety symptoms and insomnia symptom severity. Psychiatry Res;254:40-7. doi:10.1016/j.psychres.2017.04.028

13. Koh D (2020): Occupational risks for COVID-19 infection. Occup Med (Lond);70(1):3-5. doi:10.1093/occmed/kqaa036

14. Lai J, Ma S, Wang Y, Cai Z, Hu J, et al (2020): Factors Associated With Mental Health Outcomes Among Health Care Workers Exposed to Coronavirus Disease 2019. JAMA Netw open; 3(3):e203976-e203976. doi:10.1001/ jamanetworkopen.2020.3976

15. Lancet $\mathrm{T}$ (2020):COVID-19: too little, too late? Lancet (London, England);395(10226):755. doi:https://doi.org/10.1016/S01406736(20)30522-5

16. Liu N, Zhang F, Wei C, Jia Y, Shang Z, et al (2020): Prevalence and predictors of PTSS during COVID-19 outbreak in China hardest-hit areas: Gender differences matter. Psychiatry Res;287(112921):1-7. doi:10.1016/j. psychres.2020.112921

17. Liu N, Zhang F, Wei C, Jia Y, Shang Z, et al. (2020): Prevalence and predictors of PTSS during COVID-19 outbreak in China hardesthit areas: Gender differences matter. Psychiatry Res; 287(112921):1-7. doi:https://doi. org/10.1016/j.psychres.2020.112921

18. Morin CM, Belleville G, Bélanger L and Ivers H (2011): The Insomnia Severity Index : Psychometric Indicators to Detect Insomnia Cases and Evaluate Treatment Response. Sleep;34(5):18-20.

19. Qi J, Xu J, Li B, Huang J S, Yang Y, et al. 
(2020): The Evaluation of Sleep Disturbances for Chinese Frontline Medical Workers under the Outbreak of COVID-19. Sleep Med;72:1-4. doi: https://doi.org/10.1016/j.sleep.2020.05.023

20. Qiu D, Yu Y, Li R, Li Y and Xiao S (2020): Prevalence of sleep disturbances in Chinese healthcare professionals : a systematic review and meta-analysis. Sleep Breath;67:258-66. doi:10.1016/j.sleep.2019.01.047

21. Soni M, Curran VH, and Kamboj SK (2013): Neurobiology of Learning and Memory Identification of a narrow post-ovulatory window of vulnerability to distressing involuntary memories in healthy women. Neurobiol Learn Mem;104:32-8. doi:10.1016/j. nlm.2013.04.003

22. Tan BY, Chew NW, Lee G K, Jing M, Goh Y, et al. (2020): Psychological Impact of the COVID-19 Pandemic on Health Care Workers in Singapore. Ann Intern Med;10(7326):M20-1083. doi:https://doi.org/10.7326/M20-1083

23. Taylor DJ, Pruiksma KE, Hale WJ, Kelly K, Maurer D, et al (2016): Prevalence, Correlates, and Predictors of Insomnia in the US Army prior to Deployment. Sleep;39(10):1795-806. doi:https://doi.org/10.5665/sleep.6156

24. Walton M, Murray E and Christian MD (2020): Mental health care for medical staff and affiliated healthcare workers during the COVID-19 pandemic. Eur Hear J Acute Cardiovasc Care; 9(3):241-7. doi:10.1177/2048872620922795

25. Weathers FW, Litz BT, Keane TM, Palmieri PA, Marx BP et al. (2013): The PTSD checklist for dsm-5 (pcl-5). The National Center for PTSD. Available at: www.ptsd.va.gov.

26. Wright KM, Britt TW, Bliese PD, Adler AB, Picchioni D and Moore D (2011): Insomnia as Predictor Versus Outcome of PTSD and Depression Among Iraq Combat Veterans. J Clin Psychol;67(12):1240-58. doi:10.1002/ jclp. 20845

27. Xiang Y, Ma X, Cai Z J, Li SR., Xiang YQ, et al.(2008): The Prevalence of Insomnia , Its Sociodemographic and Clinical Correlates, and Treatment in Rural and Urban Regions of Beijing, China: A General Population- Based Survey. Sleep;31(12):1655-62. doi:https://doi. org/10.1093/sleep/31.12.1655

28. Yin Q, Sun Z, Liu T, Ni X, Deng X, et al (2019): Posttraumatic stress symptoms of health care workers during the corona virus disease 2019. Clin Psychol Psychother; 27:384-95. doi:10.1002/cpp.2477

29. Zhang C, Yang L, Liu S, Ma S, Wang Y, et al. (2020a): Survey of Insomnia and Related Social Psychological Factors Among Medical Staff Involved in the 2019 Novel Coronavirus Disease Outbreak. Front. Psychiatry;11(306):1-9. doi:10.3389/fpsyt.2020.00306

30. Zhang W, Wang K, Yin L, Zhao WF, Xue Q, et al (2020b): Mental Health and Psychosocial Problems of Medical Health Workers during the COVID-19 Epidemic in China. Psychother Psychosom;100053(45):1-9. doi:10.1159/000507639 
